\title{
Numerical study of nonequilibrium gas flow in a microchannel with a ratchet surface
}

\author{
Lianhua Zhu and Zhaoli Guo* \\ State Key Laboratory of Coal Combustion, School of Power and Energy Engineering, Huazhong University of Science and Technology, \\ Wuhan, 430074, China
}

(Received 31 July 2016; revised manuscript received 29 January 2017; published 23 February 2017)

\begin{abstract}
The nonequilibrium gas flow in a two-dimensional microchannel with a ratchet surface and a moving wall is investigated numerically with a kinetic method [Guo et al., Phys. Rev. E 91, 033313 (2015)]. The presence of periodic asymmetrical ratchet structures on the bottom wall of the channel and the temperature difference between the walls of the channel result in a thermally induced flow, and hence a tangential propelling force on the wall. Such thermally induced propelling mechanism can be utilized as a model heat engine. In this article, the relations between the propelling force and the top wall moving velocity are obtained by solving the Boltzmann equation with the Shakhov model deterministically in a wide range of Knudsen numbers. The flow fields at both the static wall state and the critical state at which the thermally induced force cancels the drag force due to the active motion of the top wall are analyzed. A counterintuitive relation between the flow direction and the shear force is observed in the highly rarefied condition. The output power and thermal efficiency of the system working as a model heat engine are analyzed based on the momentum and energy transfer between the walls. The effects of Knudsen number, temperature difference, and geometric configurations are investigated. Guidance for improving the mechanical performance is discussed.
\end{abstract}

DOI: 10.1103/PhysRevE.95.023113

\section{INTRODUCTION}

In rarefied gas, the inhomogeneity of temperature field can lead to gas motion, i.e., the so-called thermally induced flows [1]. The flows further exert force on objects immersed in the gas or on the confining walls. Such force can be utilized as the propelling power in some applications. A well-known application is the Crookes radiometer [2], also known as light mill. Using a Crookes radiometer, the radiation intensity is indicated by the rotation speed of the mounted vanes on a spindle enclosed in an evacuated glass bulb. The rotation of the vanes is driven by the rarefied gas flow in the bulb due to the temperature difference across the vanes. Thermally induced force can also be produced in microdevices working under regular atmosphere condition due to the nonequilibrium effect, and have found their applications in many microelectromechanical systems (MEMS) devices. For example, in an atomic force microscope (AFM), the microcantilever will experience a force if its surface temperature is different from the sample [3].

Usually, thermally induced force due to nonequilibrium effects in a gas system can be classified into several types [4] according to the origins of the flows. In the examples of Crookes radiometer and AFM, the dominant force are the so-called radiometric force and Knudsen force, respectively, both of which direct across the vanes or the microcantilevers. The radiometric force is the force exerted on a plate with differently heated sides placed in a chamber in rarefied condition. While the Knudsen force is the force exerted on two planar surfaces (usually a smaller one and a big one) kept at different temperatures separated by a distance comparable to the mean free path of the gas. The two types of force have been investigated by theoretical, experimental, and numerical approaches [4-8].

\footnotetext{
*zlguo@ hust.edu.cn
}

Another significantly different but less studied thermally induced force is the one caused by the thermal creep flow, which develops at the surface of a wall with nonuniform temperature [1]. Although such flows have been widely studied since Maxwell's seminal work from 1879 [9], the shear force induced by the flow near the wall according to the third Newton's law has drawn litter interest [4]. Note that this kind of force is fundamentally different from the radiometric force and the Knudsen force. The radiometric force and the Knudsen force are primarily caused by the pressure difference between the opposite sides, hence they act across the solid wall, while the force associated with the thermal creep flow is essentially the shear stress acting along the solid wall. Moreover, unlike the two other kinds of force, the thermal creep flow generated force can only be induced near a wall with nonuniform temperature. In addition, the shear force induced by the thermal creep flow is much weaker than the radiometric force and the Knudsen force under the same imposed temperature difference [4]. This is most likely the reason why it is less studied than the other two. However, as the direction of the shear force is along the wall, the thermally induced force does have potential advantages in some specific applications. Recent studies show that a surface temperature gradient is not necessary to produce such shear force. For instance, Donkov et al. [10] observed that a thermal creep flow and the corresponding shear force can be induced in a channel with a hot plain surface and a cold ratchet surface structured with diffusive-specular reflective segments [see Fig. 1(a)]. The observation motivated Baier et al. [11] to propose a heat energy harvesting device based on this phenomenon. A parametric study was further conducted in the free molecular regime based on the collisionless Boltzmann equation [11]. Würger [12] also theoretically estimated the thermal creep velocity and the shear stress generated in the configuration, and he claimed that the thermal creep flow is the driving mechanism of the self-propelling Leidenfrost droplets over a ratchet surface which has been experimentally observed [13-15]. It should be 


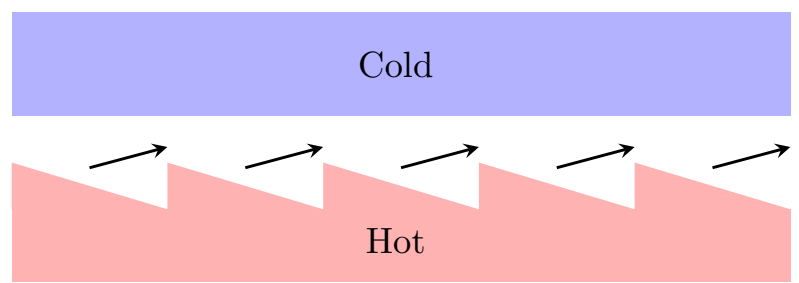

(a)

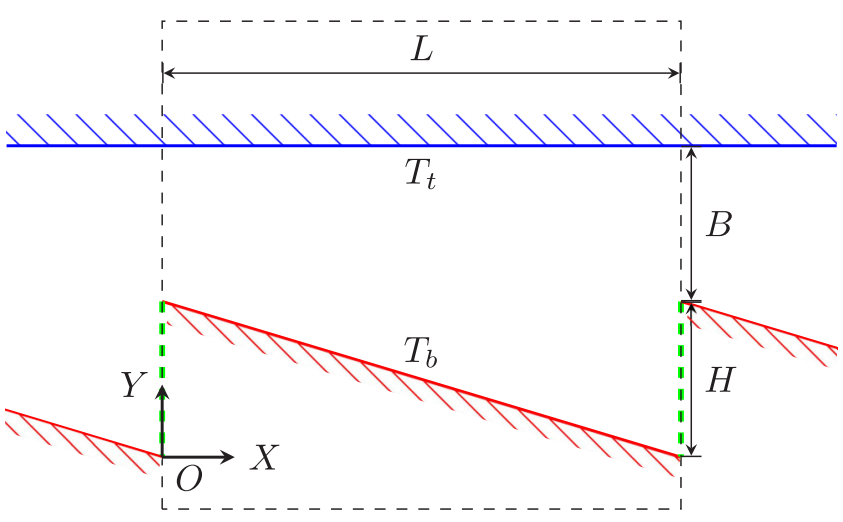

(b)

FIG. 1. (a) Channel with ratchet surface. The top and bottom walls are maintained at different temperatures. (b) One segment (in the dashed line rectangle) of the periodical structures. The thick blue and red solid lines with hatched patterns represent diffusive solid walls maintained at different temperatures $T_{b}$ and $T_{t}$, respectively. The thick dashed green lines represent specularly reflective solid walls.

noted that in his analysis, the gas-wall interaction property is assumed to be homogeneous on the ratchet surface. However, a later study by Hardt et al. [16] on a similar geometry using the direct simulation Monte Carlo (DSMC) method shows that if the ratchet surface is diffusive everywhere, the shear force is not sufficient to propel the droplets.

In most of the previous studies [10,12,16,17], the ratchet surface of the channel is at rest with respect to the opposite surface, as the primary interest in these studies is the mass flow rate across the channel when it works as an Knudsen pump. However, if the force produced by the thermally induced flow in such geometry is to be applied as the self-propelling power of microdevices or in a microheat engine [11], the two surfaces may move relative to each other. In Ref. [11], the shear stress as a function of the surface velocity was studied, and it is found the shear stress changes linearly with the surface speed. However, their numerical analysis is limited to free molecular limit only. In practical applications, the system should work in the transition regime to maximize the net power output [10]. Therefore, it is necessary to study the thermally induced flow with movable surfaces in such configurations in a wide range of operating conditions. However, it is a challenging task to simulate such low-speed thermally induced nonequilibrium flows, which is difficult for the widely used DSMC method, particularly in slip and near-continuum regimes. On the other hand, analysis based on the Navier-Stokes equations is limited to near-continuum flows [17].
In this work, we perform a numerical study on the two-dimensional nonequilibrium gas flow and investigate the corresponding shear force (or tangential force) in a ratchet channel between two walls with different temperatures and velocities. The mechanical performance including the output power and thermal efficiency of the system when working as a heat engine are analyzed. The effects of the Knudsen number, the geometric configuration, and the temperature configuration are investigated. The numerical method employed here is the recently developed discrete unified gas-kinetic scheme (DUGKS) based on the Boltzmann-Shakhov equation [18,19], which applies to the whole range of Knudsen number, and is particularly efficient for low speed near continuum flows.

The remainder of the paper is organized as follows. In Sec. II, we first describe the problem and the major assumptions. Then, in Sec. III, the governing equations are presented. In Sec. IV, we first use a test case to verify our method against the DSMC method, and then the numerical results are presented and analyzed. Finally, conclusions are made in Sec. V.

\section{STATEMENT OF THE PROBLEM}

The problem considered is a unit cell of an infinite channel shown in Fig. 1(a). The configuration and geometrical parameters are illustrated in Fig. 1(b). The top plate is placed horizontally and is assumed to be purely diffusive. It maintains at a constant temperature $T_{t}$ and moves horizontally with a constant velocity $U_{w}$. The bottom wall is a stationary ratchet wall with a saw-tooth-like structure. Each segment of the structure on the ratchet wall is further composed of a segment of an inclined diffusive wall and a segment of a vertical specular wall [see in Fig. 1(b)]. The inclined wall maintains at a constant temperature $T_{b}$ which is different from $T_{t}$. The width and height of the saw-tooth are $L$ and $H$, respectively. The distance from the tip of the saw-tooth to the top plate is $B$. Due to the periodic structures in this configuration, the flow pattern is also periodical in the horizontal direction with a period length of $L$. Therefore, we only consider one period of the channel as illustrated in Fig. 1(b). The gas in the channel is argon with a temperature-dependent viscosity as [20]

$$
\mu=\mu_{\text {ref }}\left(\frac{T}{T_{\text {ref }}}\right)^{\omega},
$$

where $\mu_{\text {ref }}$ is the reference viscosity at the reference temperature $T_{\text {ref }}$, and $\omega$ is a constant dependent on the specific molecular interaction model. For the currently considered argon gas, $\omega$ is set to be 0.81 which is consistent with the variable hard-sphere (VHS) model of argon gas [20]. The reference viscosity is also determined by the VHS model [20],

$$
\mu_{\text {ref }}=\frac{15 \rho \lambda \sqrt{2 \pi R T_{\text {ref }}}}{2(7-2 \omega)(5-2 \omega)},
$$

where $\rho$ is density of the gas, and $\lambda$ is the mean free path (mfp). The mfp is inversely proportional to $\rho$ by [20]

$$
\lambda=\frac{m}{\sqrt{2} \rho \pi d^{2}},
$$

where $m=6.63 \times 10^{-26} \mathrm{~kg}$ and $d=4.17 \times 10^{-10} \mathrm{~m}$ are the mass and hard-sphere diameter of the argon gas molecular. The 
Knudsen number of the system is defined as $\mathrm{Kn}=\lambda /(B+$ $H / 2)$ [16].

\section{GOVERNING EQUATIONS}

The governing equation is the Boltzmann equation with the Shakhov collision model [21],

$$
\frac{\partial f}{\partial t}+\boldsymbol{\xi} \cdot \nabla f=\Omega^{S}(f)
$$

where $f=f(\boldsymbol{\xi}, \boldsymbol{x}, t)$ is the velocity distribution function of particles with velocity $\boldsymbol{\xi}$ at time $t$ and position $\boldsymbol{x} . \Omega^{S}(f)$ is the Shakhov collision term given by

$$
\Omega^{S}(f)=\frac{f^{S}-f}{\tau},
$$

where $\tau$ is the relaxation time. The modified equilibrium distribution function is defined as

$$
\begin{aligned}
f^{S} & =f^{M}\left[1+(1-\operatorname{Pr}) \frac{\boldsymbol{c} \cdot \boldsymbol{q}}{5 P R T}\left(\frac{c^{2}}{R T}-5\right)\right], \\
\text { with } \quad f^{M} & =\frac{\rho}{(2 \pi R T)^{3 / 2}} \exp \left(-\frac{c^{2}}{2 R T}\right),
\end{aligned}
$$

where Pr is the Prandtl number and $\boldsymbol{c}=\boldsymbol{\xi}-\boldsymbol{U}$ is the peculiar velocity with $\boldsymbol{U}$ being the fluid velocity. For a monatomic gas, $\operatorname{Pr}$ is equal to $2 / 3$. The macroscopic variables such as the density $\rho$, velocity $\boldsymbol{U}$, temperature $T$, stress tensor $\boldsymbol{p}$, and heat flux $\boldsymbol{q}$ can be calculated from the moments of the distribution function,

$$
\begin{aligned}
& \rho=\int f d \boldsymbol{\xi}, \quad \rho \boldsymbol{U}=\int \boldsymbol{\xi} f d \boldsymbol{\xi}, \quad \rho E=\frac{1}{2} \int \xi^{2} d \boldsymbol{\xi}, \\
& \boldsymbol{q}=\frac{1}{2} \int \boldsymbol{c} c^{2} f d \boldsymbol{\xi}, \quad \boldsymbol{p}=\int \boldsymbol{\xi} \boldsymbol{\xi} f d \boldsymbol{\xi},
\end{aligned}
$$

where $\rho E=1 / 2 \rho U^{2}+C_{v} T$ is the total energy with $C_{v}$ being the heat capacity $[(3 / 2) R$ for monatomic gases]. The pressure is related to the density and temperature by $P=\rho R T$, and the relaxation time $\tau$ is related to the viscosity as $\tau=\mu / P$.

For the two-dimensional flow considered here, the dependence of distribution function on the $z$ component of $\xi$, i.e., $\xi_{z}$ can be eliminated from the governing equation by introducing the following reduced distribution functions:

$$
\Phi=\left[\begin{array}{l}
g \\
h
\end{array}\right]=\int_{-\infty}^{\infty}\left[\begin{array}{c}
1 \\
\xi_{z}^{2}
\end{array}\right] f(\boldsymbol{\xi}, \boldsymbol{x}, t) d \xi_{z} .
$$

Integrating Eq. (4) over the range of $[-\infty, \infty]$ with respect to $\xi_{z}$, and noting that $\partial \Phi / \partial z=0$ leads to

$$
\frac{\partial \Phi}{\partial t}+\xi_{x} \frac{\partial \Phi}{\partial x}+\xi_{y} \frac{\partial \Phi}{\partial y}=-\frac{1}{\tau}\left[\Phi-\Phi^{S}\right] \equiv \Omega
$$

in two-dimensional space, with $\Phi^{S}=\left[g^{S}, h^{S}\right]^{T}$ and

$$
\begin{aligned}
g^{S} & =g^{M}\left[1+(1-\operatorname{Pr}) \frac{\boldsymbol{c} \cdot \boldsymbol{q}}{5 P R T}\left(\frac{c^{2}}{R T}-4\right)\right], \\
h^{S} & =g^{M}\left[1+(1-\operatorname{Pr}) \frac{\boldsymbol{c} \cdot \boldsymbol{q}}{5 P R T}\left(\frac{c^{2}}{R T}-2\right)\right] R T, \\
g^{M} & =\frac{\rho}{2 \pi R T} \exp \left(-\frac{c^{2}}{2 R T}\right) .
\end{aligned}
$$

Now, all the vector variables have only two components, such as $\boldsymbol{x}=[x, y]$ and $\boldsymbol{\xi}=\left[\xi_{x}, \xi_{y}\right]$.

The governing equation can be written in a nondimensional form as

$$
\begin{aligned}
\frac{\partial \hat{\Phi}}{\partial \hat{t}}+\hat{\xi}_{x} \frac{\partial \hat{\Phi}}{\partial \hat{x}}+\hat{\xi}_{y} \frac{\partial \hat{\Phi}}{\partial \hat{y}}= & \frac{8}{5 \sqrt{\pi}} \frac{1}{(\hat{B}+\hat{H} / 2) \mathrm{Kn}} \\
& \times \hat{\rho} \hat{T}^{1-\omega}\left[\hat{\Phi}-\hat{\Phi}^{S}\right],
\end{aligned}
$$

where

$$
\begin{aligned}
& \hat{g}^{S}=\hat{g}^{M}\left[1+\frac{4}{5}(1-\operatorname{Pr}) \frac{\hat{\boldsymbol{c}} \cdot \hat{\boldsymbol{q}}}{\hat{\rho} \hat{T}^{2}}\left(\frac{2 \hat{c}^{2}}{\hat{T}}-4\right)\right], \\
& \hat{h}^{S}=\hat{g}^{M}\left[1+\frac{4}{5}(1-\operatorname{Pr}) \frac{\hat{\boldsymbol{c}} \cdot \hat{\boldsymbol{q}}}{\hat{\rho} \hat{T}^{2}}\left(\frac{2 \hat{c}^{2}}{\hat{T}}-2\right)\right] \frac{\hat{T}}{2}, \\
& \hat{g}^{M}=\frac{\hat{\rho}}{\pi \hat{T}} \exp \left(-\frac{\hat{c}^{2}}{\hat{T}}\right),
\end{aligned}
$$

and the nondimensional variables are defined by

$$
\begin{aligned}
& \hat{x}=x / L, \quad \hat{y}=y / L, \quad \hat{\boldsymbol{\xi}}=\boldsymbol{\xi} / \sqrt{2 R T_{0}}, \\
& \hat{\rho}=\rho / \rho_{0}, \quad \hat{\boldsymbol{U}}=\boldsymbol{U} / \sqrt{2 R T_{0}}, \quad \hat{\boldsymbol{p}}=\boldsymbol{p} /\left(\rho_{0} R T_{0}\right), \\
& \hat{T}=T / T_{0}, \quad \hat{\boldsymbol{q}}=\boldsymbol{q} / \rho_{0}\left(2 R T_{0}\right)^{3 / 2}, \quad \hat{g}=g /\left(\rho_{0} / R T_{0}\right), \\
& \hat{h}=h / \rho_{0}, \quad \hat{\Phi}=\left[\begin{array}{ll}
\hat{g}, & \hat{h}
\end{array}\right]^{T}, \quad \hat{\Phi}^{s}=\left[\begin{array}{ll}
\hat{g}^{s}, & \hat{h}^{s}
\end{array}\right]^{T}, \\
& \hat{B}=B / L, \quad \hat{H}=H / L, \quad \hat{t}=t /\left(L / \sqrt{2 R T_{0}}\right),
\end{aligned}
$$

where $\rho_{0}$ and $T_{0}$ are the reference density and temperature, respectively. The hats on the nondimensional variables are omitted in the following analysis.

The gas-wall interaction models have to be specified to implement the boundary conditions at the channel walls. Two kinds of gas-wall interaction models are considered for different segments of the ratchet walls. The first one is the fully diffusive wall model, where the gas molecules that hit the wall are assumed to be fully accommodated with the wall, and are reemitted from the wall with an equilibrium distribution associated with the nondimensional wall temperature $T_{w}$ and velocity $\boldsymbol{U}_{w}$,

$$
\Phi=\left[\begin{array}{c}
1 \\
T_{w} / 2
\end{array}\right] g^{M}\left(\rho_{w}, \boldsymbol{U}_{w}, T_{w}\right) \text { for } \boldsymbol{\xi} \cdot \boldsymbol{n}_{w}>0,
$$

where $\boldsymbol{n}_{w}$ is the unit normal vector of the wall pointing into the flow domain, and $g^{M}\left(\rho_{w}, \boldsymbol{U}_{w}, T_{w}\right)$ stands for the equilibrium distribution function of gas molecular with velocity $\boldsymbol{\xi}$ with the arguments of density $\rho_{w}$, temperature $T_{w}$, and macrovelocity $\boldsymbol{U}_{w}$. The density $\rho_{w}$ is determined by the impermeable wall condition, i.e.,

$$
\rho_{w}=-\frac{\int_{\boldsymbol{\xi} \cdot \boldsymbol{n}_{w}<0} g \boldsymbol{\xi} \cdot \boldsymbol{n}_{w} d \boldsymbol{\xi}}{\int_{\boldsymbol{\xi} \cdot \boldsymbol{n}_{w}>0} g^{M}\left(1, \boldsymbol{U}_{w}, T_{w}\right) \boldsymbol{\xi} \cdot \boldsymbol{n}_{w} d \boldsymbol{\xi}} .
$$

The second type of gas-wall interaction model is the specular reflective boundary condition, in which the wall is assumed to be ideally smooth and the gas molecules striking the wall will be reflected with their tangential velocity component unchanged but normal velocity component reversed, i.e.,

$$
\boldsymbol{\xi}^{r}=\boldsymbol{\xi}^{i}-2\left(\boldsymbol{\xi}^{i} \cdot \boldsymbol{n}_{w}\right) \boldsymbol{n}_{w},
$$


where the superscripts $i$ and $r$ denote the incoming and reflecting directions, respectively.

\section{RESULTS AND ANALYSES}

The numerical scheme used to solve the kinetic equation is the discrete unified gas-kinetic scheme (DUGKS) [19,22], which is a finite-volume scheme for the discrete velocity Boltzmann model equation [20]. Compared with the stochastic DSMC method, the deterministic nature of DUGKS makes it more efficient for low-speed flow problem in this study. Moreover, the DUGKS is also efficient for near continuum flow due to the special construction of distribution flux at cell interfaces [19]. The algorithm of the DUGKS is briefly described in the Appendix.

We now present the numerical results for various operating conditions $\left(\mathrm{Kn}, T_{t}, T_{b}\right)$ and geometry configurations ( $B$, $H$ ). Unless stated otherwise, the base parameters are given as follows. The nondimensional geometric parameters are $H=0.3$ and $B=0.3$. The wall temperatures are $T_{t}=0.75$ and $T_{b}=1.25$. The top wall is at rest, i.e., $U_{w}=0$. In all of the computations below, the Courant-Friedrichs-Lewy (CFL) number defined as $\eta=\Delta t \min [(|\boldsymbol{U}|+|\boldsymbol{\xi}|) / \Delta x]$ is set to be 0.8 where $\Delta x$ measures the local cell size [22]. The physical space is discretized with an unstructured mesh with 5623 triangular cells. The smallest cells are distributed in the regions near the tip of the ratchet and the upper wall in order to capture the large gradients and to compute the shear stress more accurately. Grid independence is checked by comparing the tangential force acting on the top plate using a mesh with 5632 cells and a finer one with 18220 cells. The relative difference in the values of the tangential force by using the two meshes is less than $2 \%$, indicating that the coarser mesh is fine enough to obtain mesh-independent results. The discrete velocity grids and the quadrature rules for the numerical integrations of the moments are chosen according to the Knudsen number as listed in Table. I. For small Knudsen numbers $(\mathrm{Kn}<0.1)$, the half-range GaussHermite type quadrature is appropriate since the distribution functions deviate only slightly from the Maxwell-Boltzmann equilibrium distribution function. For larger Knudsen numbers $(\mathrm{K} n \geqslant 0.1)$, we use the compound Newton-Cotes quadrature since the distribution function can be highly nonequilibrium.
TABLE I. Numbers of quadrature points in the velocity space and quadrature types at different Knudsen numbers $(\mathrm{Kn})$ used in the DUGKS method.

\begin{tabular}{llr}
\hline \hline Kn & \multicolumn{1}{c}{ Quadrature type } & No. of points \\
\hline 0.001 & Half-range Gauss-Hermite & $8 \times 8$ \\
0.01 & Half-range Gauss-Hermite & $12 \times 12$ \\
0.05 & Half-range Gauss-Hermite & $20 \times 20$ \\
$0.1-0.5$ & Compound Newton-Cotes & $41 \times 41$ \\
$0.7-1$ & Compound Newton-Cotes & $61 \times 61$ \\
$2-5$ & Compound Newton-Cotes & $81 \times 81$ \\
10 & Compound Newton-Cotes & $201 \times 201$ \\
\hline \hline
\end{tabular}

The nondimensional discrete velocity points are distributed uniformly in the range of $[-4,4] \times[-4,4]$ when using the compound Newton-Cotes quadrature. The velocity space grid independence of the numerical results has also been verified. For example, at $\mathrm{Kn}=0.5$, the relative difference of the tangential force acting on the top wall using the velocity grid sizes of $41 \times 41$ and $61 \times 61$ is less than $0.3 \%$. As the DUGKS is an explicit method, the flow is assumed to have reached steady states if the average relative change of the velocity fields in two successive steps is less than $10^{-8}$.

\section{A. Validation}

Before presenting the numerical results, we first validate our numerical method against DSMC method at $\mathrm{Kn}=0.1$ and $U_{w}=0.01$. The DSMC code used here is the open source parallel DSMC solver, dsmcFoam [23]. Both the DSMC and DUGKS solvers use the same mesh with 5632 cells. A total of 112640 particles are used in the DSMC simulation with an average of 20 particles residing in each cell initially.

The temperature and flow fields predicted by both methods are shown in Fig. 2. It can be observed that the DUGKS results agree well with the DSMC predictions. Due to the low speed of the flows, the statistic noises can be clearly observed from the streamlines of the DSMC results, especially in the vortex region near the bottom corner, where the velocity magnitude is very small compared to the thermal velocity. To compare the two results quantitatively, we present the shear stress and normal heat flux distributions on the top wall in Fig. 3, and present the velocity and temperature profiles across the throat

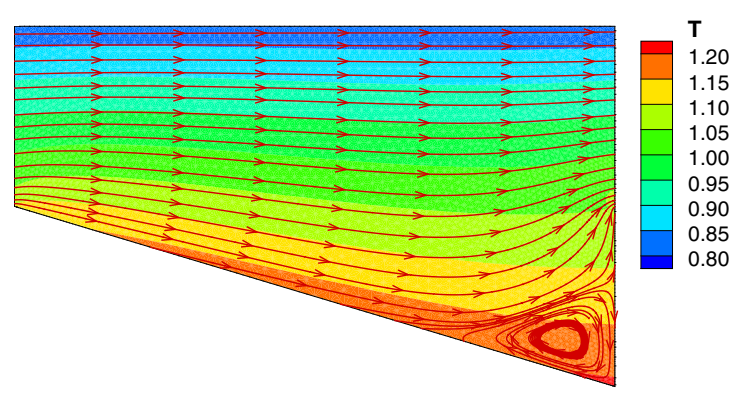

(a) DUGKS

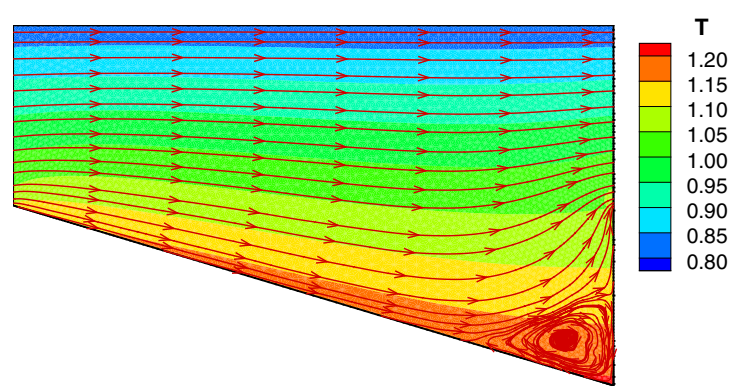

(b) DSMC

FIG. 2. Isotherms and streamlines of the validation case $\left(\mathrm{Kn}=0.1, U_{w}=0.01\right)$. (a) Results obtained by the DUGKS. (b) Results obtained by DSMC method. 


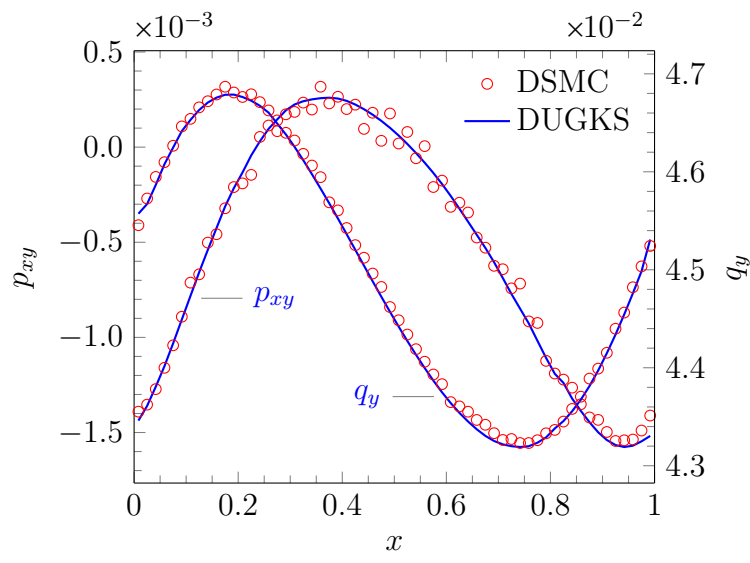

FIG. 3. Shear stress $p_{x y}$ and normal heat flux $q_{y}$ along the top wall for the validation case $\left(\mathrm{Kn}=0.1, U_{w}=0.01\right)$ predicted by both DSMC method and the DUGKS.

of the channel in Fig. 4. From those results, we can see the DUGKS results match the DSMC results quite well, despite the statistic noises in the DSMC results.

Given the good agreement between the two methods, we are confident that the DUGKS can be faithfully used to study the thermally induced flow problems. We would like to mention the computational costs of the two methods. Both of the DSMC and DUGKS solvers were run with 12 message passing interface (MPI) processes on an Intel Xeon E5-2680v3 CPU. The DSMC code took about $160 \mathrm{~h}$ get the steady results, while the DUGKS code took only $4 \mathrm{~h}$. This contrast clearly demonstrates for such thermally induced low-speed flows the deterministic solver is significantly faster than the DSMC solver.

\section{B. Relation between shear stress and wall velocity}

In this subsection, we analyze the tangential force acting on the top wall at different Knudsen numbers and its relation with the top wall velocity. The tangential force $F$ is calculated by integrating the shear stress along the top plate assuming a unit length in the $Z$ direction. The tangential force exerted on

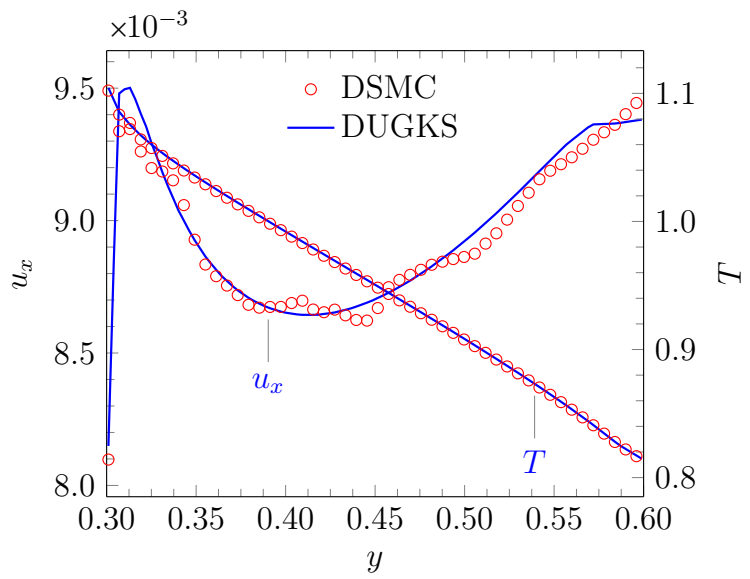

FIG. 4. Temperature $T$ and horizontal velocity $u_{x}$ profiles across the throat of the channel for the validation case $\left(\mathrm{Kn}=0.1, U_{w}=\right.$ 0.01) predicted by both DSMC method and the DUGKS.

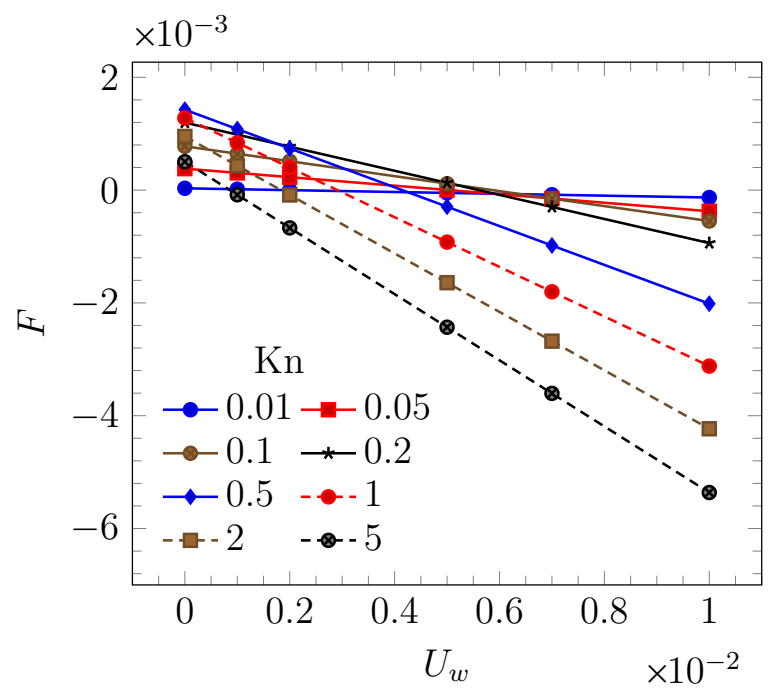

FIG. 5. Tangential force acted on the top wall against wall velocity at different Knudsen numbers ranging from 0.01 to 5 .

the top wall is a combined effect of thermally induced flow and the active motion of the top wall. The thermally induced flow is from left to right; hence it induces a rightward tractive force on the wall, whereas the gas will exert a leftward drag force in response to the active motion of the wall. Therefore, it is expected that $F$ will direct to the right as $U_{w}=0$, and its magnitude will decrease with the increasing of $U_{w}$, even reverse its direction if $U_{w}$ is large enough.

The dependence of $F$ on $U_{w}$ can be analyzed from the moment exchange of the molecules hitting on and leaving from the wall. The force exerting on the top wall by the incident molecules is

$$
\boldsymbol{F}^{-}=-\int_{\xi \cdot \boldsymbol{n}<0}(\boldsymbol{\xi} \cdot \boldsymbol{n}) \boldsymbol{\xi} g^{-}(\boldsymbol{\xi}) d \boldsymbol{\xi},
$$

where $g^{-}(\xi)$ is the reduced distribution function of the incoming molecules with incident velocity $\boldsymbol{\xi}$, and $\boldsymbol{n}$ is the inward unit normal vector pointing into the gas. On the other hand,

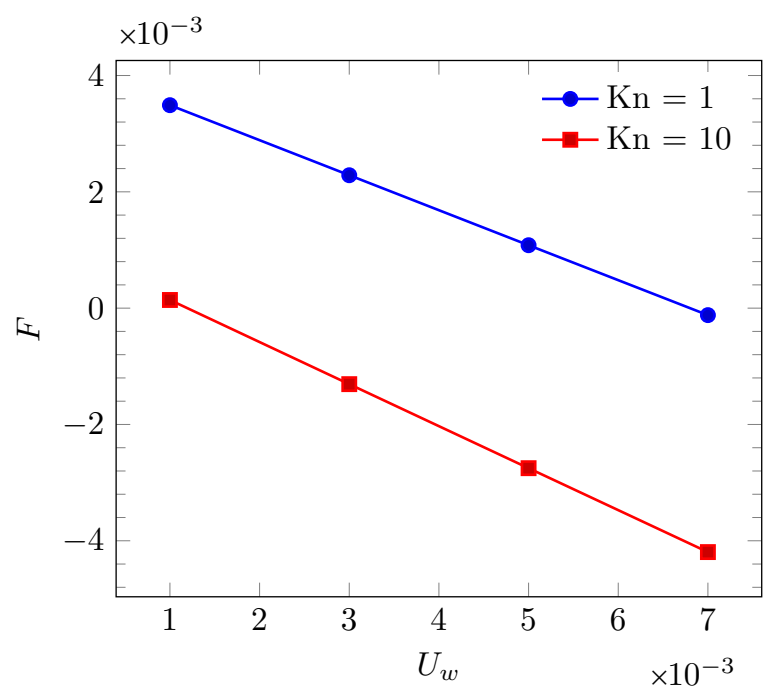

FIG. 6. Tangential force acted on the top wall against wall velocity for the case of $H=0.9$ and $B=0.1$ at $\mathrm{Kn}=1$ and $\mathrm{Kn}=10$. 


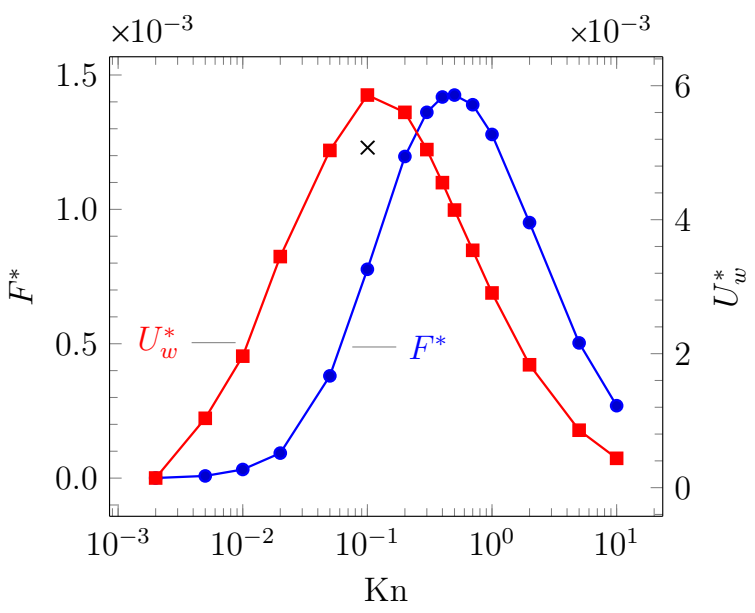

FIG. 7. Tangential force on a static wall (SWF in the body text) and shear-free wall velocity (SFV in the body text) at various Knudsen numbers.

the force exerting by the molecules leaving from the wall is

$$
\boldsymbol{F}^{+}=-\int_{\xi \cdot n>0}(\boldsymbol{\xi} \cdot \boldsymbol{n}) \boldsymbol{\xi} g^{+}(\boldsymbol{\xi}) d \boldsymbol{\xi},
$$

where $g^{+}(\xi)$ is the reduced distribution function of the outgoing molecules with velocity $\xi$. Since the wall is diffusive, $g^{+}$can be expressed as

$$
g^{+}(\boldsymbol{\xi})=g^{M}\left(\rho_{w}, u_{w}, T_{w}\right),
$$

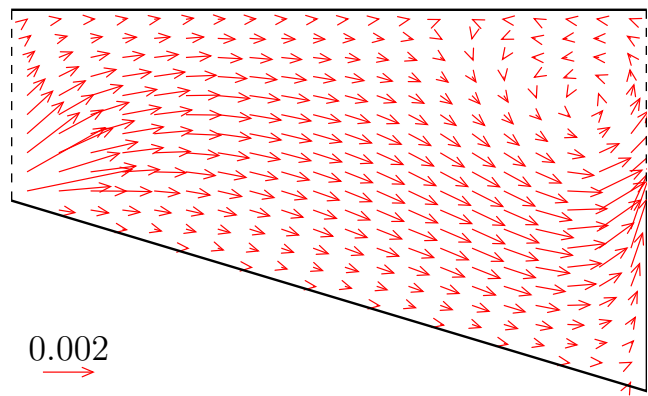

(a) $\mathrm{Kn}=0.01$

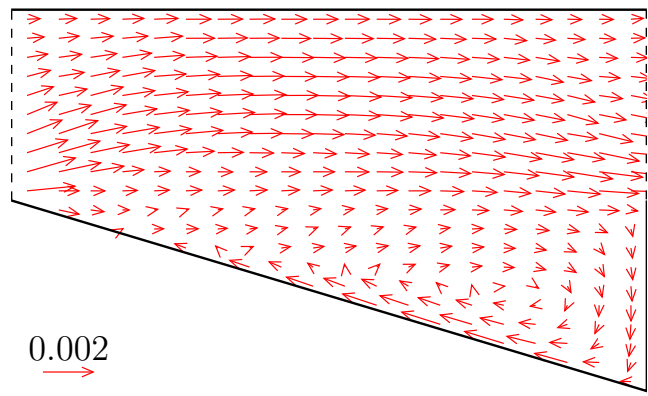

(c) $\mathrm{Kn}=0.5$ where $\rho_{w}$ is given by Eq. (15). Then a direct calculation of Eq. (18) leads to [11],

$$
\boldsymbol{F}^{+}=-\varsigma\left[\left(\pi R T_{w} / 2\right)^{1 / 2} \boldsymbol{n}+u_{w} \boldsymbol{t}\right],
$$

where $\varsigma=\int_{\xi \cdot n<0}|\boldsymbol{\xi} \cdot \boldsymbol{n}| g^{-}(\boldsymbol{\xi}) d \boldsymbol{\xi}$ is the molecule flux and $\boldsymbol{t}$ is the unit tangential vector. The net force on the wall is then $\boldsymbol{F}=\boldsymbol{F}^{+}+\boldsymbol{F}^{-}$. Specifically, the tangential force can be expressed as

$$
F=F_{x}^{-}-\varsigma u_{w},
$$

or in nondimensional form

$$
F\left(U_{w}\right)=F^{*}\left(1-\frac{U_{w}}{U_{w}^{*}}\right),
$$

where $F^{*}$ and $U_{w}^{*}$ are two parameters that may depend on the Knudsen number for a specific geometric and temperature configuration. Actually, $F^{*}$ represents the tangential force as $U_{w}=0$, i.e., the pure thermally induced force, which is termed as static wall force (SWF) in this work; $U_{w}^{*}$ represents the wall velocity at which the tangential force vanishes. It is also the terminal wall velocity when the system approaches the steady state if we assume the wall can move freely in the horizontal direction. This critical velocity is termed as shear free velocity (SFV) in this work. We note that similar concepts were also introduced in Ref. [11]. $F^{*}$ and $U^{*}$ are two important parameters which characterize the response of the system to the variation of the top wall velocity.

The result given by Eq. (22) suggests that the tangential force on the top wall is linearly dependent on the wall velocity.

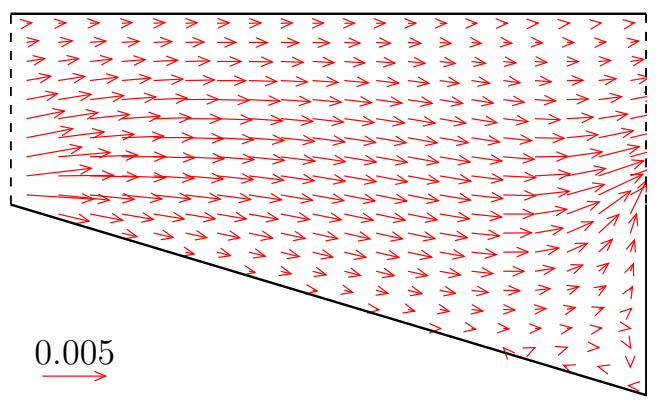

(b) $\mathrm{Kn}=0.1$

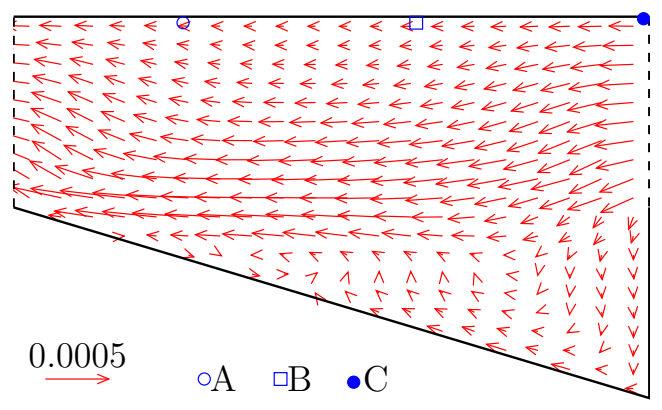

(d) $\mathrm{Kn}=10$

FIG. 8. Velocity fields with static top wall configuration, i.e., $U_{w}=0$ at different Knudsen numbers. (a) $\mathrm{Kn}=0.01$. (b) $\mathrm{Kn}=0.1$. (c) $\mathrm{Kn}=1$. (d) $\mathrm{Kn}=10$. Data have been interpolated to structured grids for better presentation. 


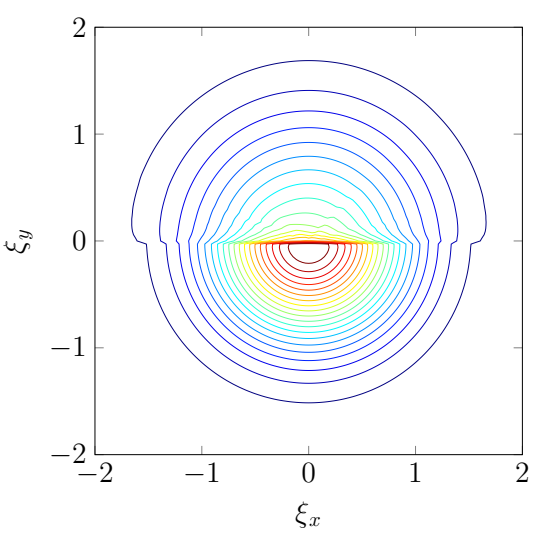

(a) Location A

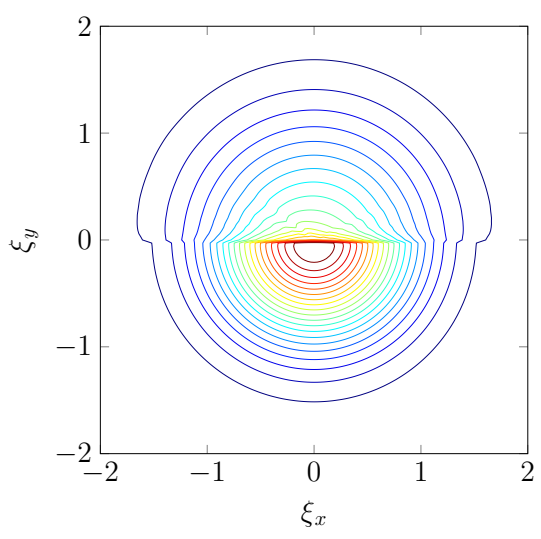

(b) Location B

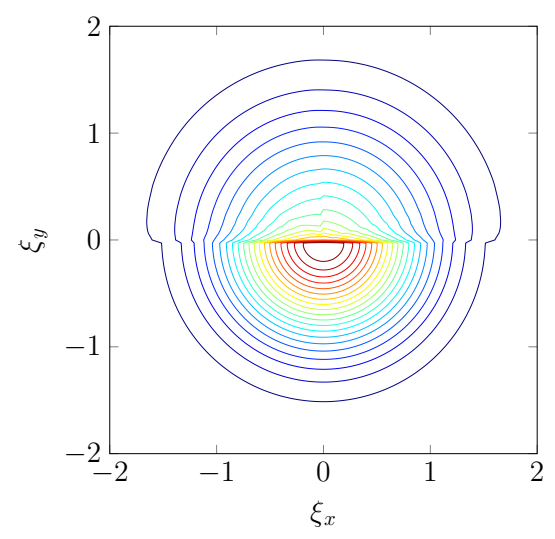

(c) Location $\mathrm{C}$

FIG. 9. Contours of the distribution function at three points near the top wall [marked in Fig. 8(d)] when Kn $=10$.

Actually, in Ref. [11] this dependence has been derived analytically in the free-molecule case (i.e., $\mathrm{Kn} \rightarrow \infty$ ) for a similar configuration. But at finite $\mathrm{Kn}$, it is difficult, if not impossible, to derive the exact values of $F^{*}$ and $U_{w}^{*}$.

In Fig. 5, the profiles of $F$ against $U_{w}$ at different values of Kn are shown. The linear relationship between $F$ and $U_{w}$ is clearly shown. It should be noted that even though these numerical results are obtained with a specific value of $H$ and $B$, the linearity of the relationship should still hold at other configurations of $H$ and $B$ since the above analytical analysis does not make assumption on the value of $H$ and $B$. For example, for an additional case of $H=0.9$ and $B=0.1$, the $F \sim U_{w}$ relations obtained from numerical simulations at $\mathrm{Kn}=0.1$ and 1 presented in Fig. 6 are also clearly linear.

It is also evident from Fig. 5 that the linearity of the relation between $F$ and $U_{w}$ depends on $\mathrm{Kn}$. In Fig. 7, we show the dependencies of $F^{*}$ and $U_{w}^{*}$ on the Knudsen number. It can be seen both $F^{*}$ and $U_{w}^{*}$ vanish in the continuum and free molecular limits, and their maximum values appear at around $K n=0.5$ and 0.1 , respectively. It is interesting that in the study of the thermally induced flow in a similar ratchet channel with different boundary conditions [10], the average velocity also takes its maximum value at $\mathrm{Kn} \approx 0.1$, but the tangential force reaches its maximum after around $\mathrm{Kn}>10$. This difference can be attributed to the different wall configurations. In the present study, the vertical wall is specularly reflective, and the inclined bottom wall is diffusive reflective, while in the case studied in Ref. [10], the wall reflective properties are just the reverse.

The velocity fields with the steady wall configuration at $\mathrm{Kn}=0.01,0.1,0.5$, and 10 are shown in Fig. 8. It can be seen that the flow patterns at different Knudsen numbers are quite different. At very small $\mathrm{Kn}(\mathrm{Kn}=0.01)$, a vortex appears near the throat, and the flow is accelerated at the tip of the ratchet, whereas at large Knudsen numbers, vortices are generated in the bottom corner even though the flow is very weak there. An interesting but counterintuitive phenomenon observed from Fig. 8 is that, at smaller Knudsen numbers, the overall flow direction is from left to right, but at $\mathrm{Kn}=10$ the flow direction is reversed. This phenomenon can be explained by the more pronounced effect of the specular reflective vertical wall in higher rarefied condition. A more interesting observation is that the SWF is still rightward even though the flow direction has changed to the leftward near the top wall at the case

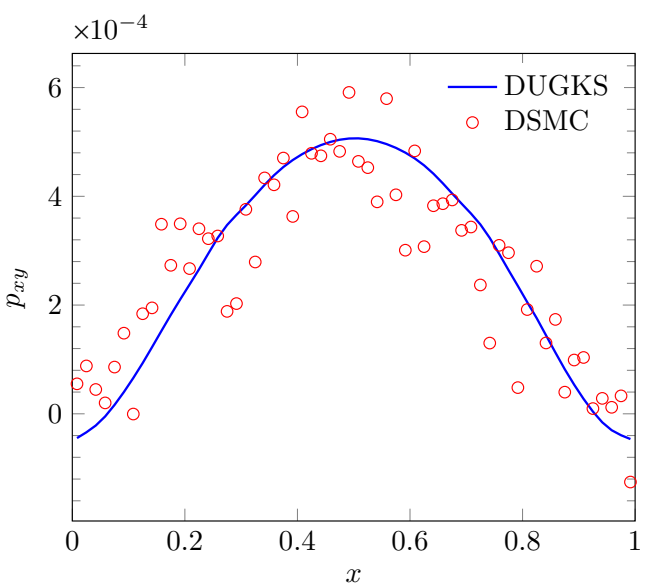

(a)

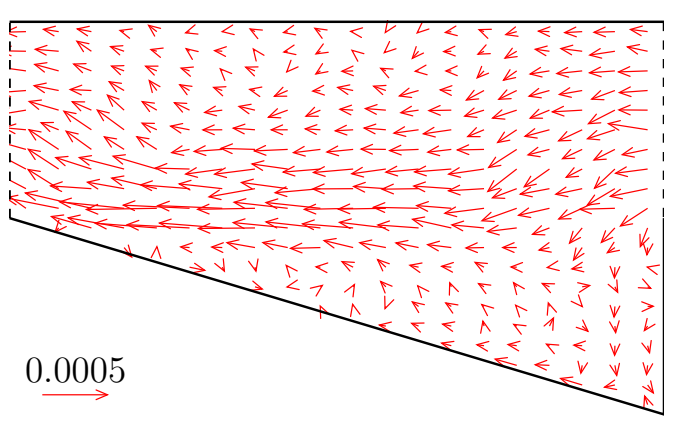

(b)

FIG. 10. Results of the case of $\mathrm{Kn}=10, U_{w}=0$ using DSMC method. (a) Shear stress distribution at the top wall (the DUGKS solution has been included). (b) Velocity fields. 


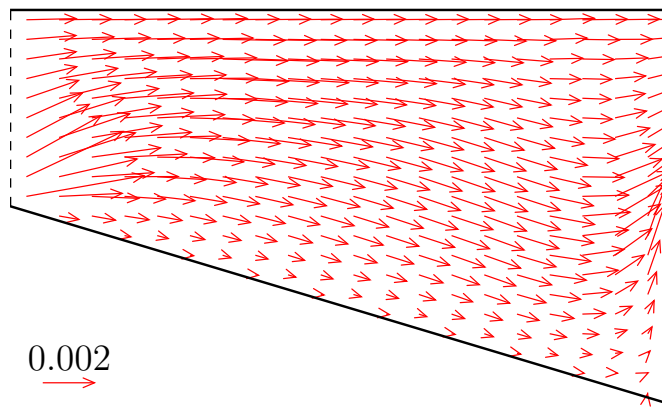

(a) $\mathrm{Kn}=0.01$

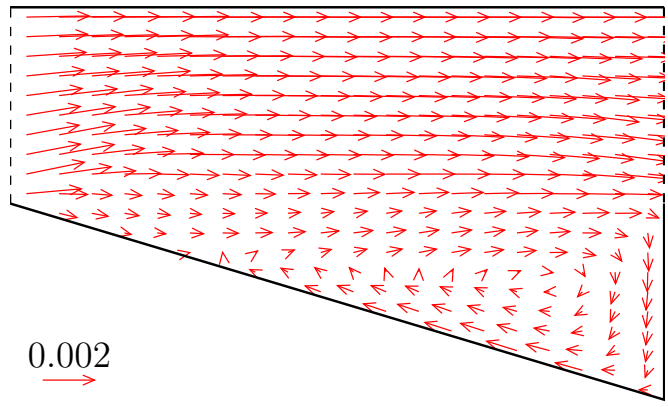

(c) $\mathrm{Kn}=1$

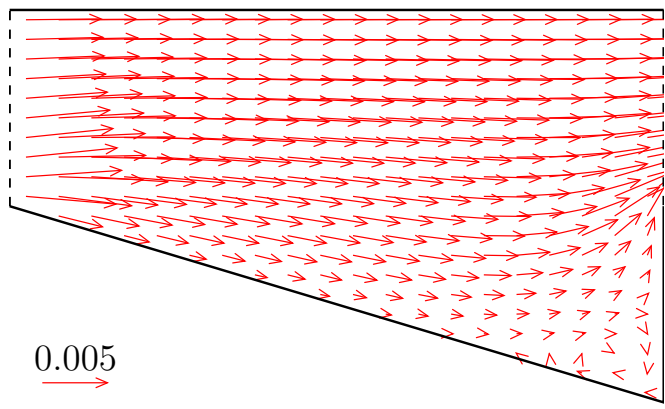

(b) $\mathrm{Kn}=0.1$

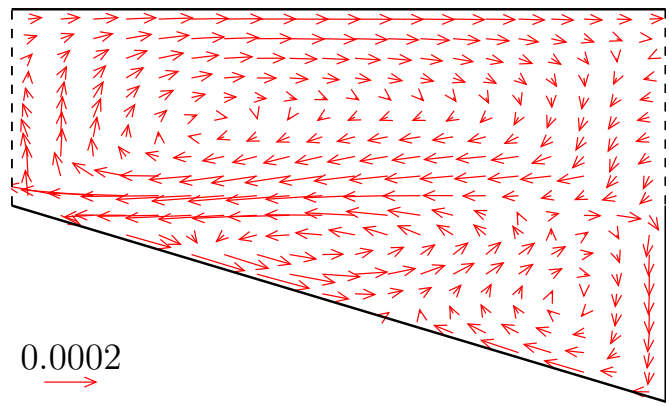

(d) $\mathrm{Kn}=10$

FIG. 11. Velocity field at shear-free state, i.e., when $F=0$ at different Knudsen numbers. (a) $\mathrm{Kn}=0.01$. (b) $\mathrm{Kn}=0.1$. (c) $\mathrm{Kn}=1$. (d) $\mathrm{Kn}=10$. Data have been interpolated to structured grids for better presentation.

of $\mathrm{Kn}=10$, which contrasts to our intuition based on the Newton's shear force law. The reason is that the distribution function near the top wall is highly nonequilibrium, which can be clearly seen from Fig. 9, where the contour plots of the distribution functions at three points near the top wall [marked in Fig. 8(d)] are presented. An independent DSMC simulation has also been conducted to corroborate this counterintuitive phenomenon. The shear force distribution along the top wall and flow field are shown in Fig. 10, from which we can see the shear force exerted on the top wall predicted by
DSMC method agrees with our results using the DUGKS despite exhibiting significant statistic noises. The flow field in Fig. 10(b) also agrees with Fig. 8(d). Particularly, the main flow direction indeed reverses from rightward to leftward, even though the force retains its direction compared with lower Knudsen number cases.

The shear-free state of the system is a critical state at which the thermal driving effect exactly balances the driving effect due to the active moving wall. This state is equivalent to the load-free state if the system works as a heat engine with ideal

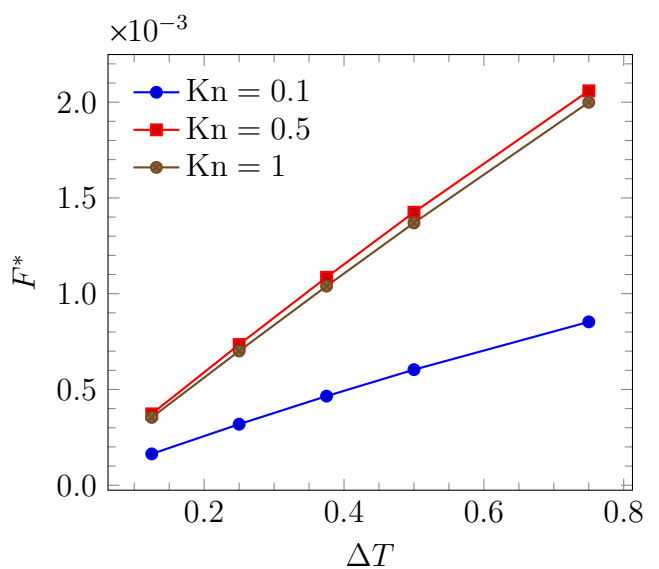

(a)

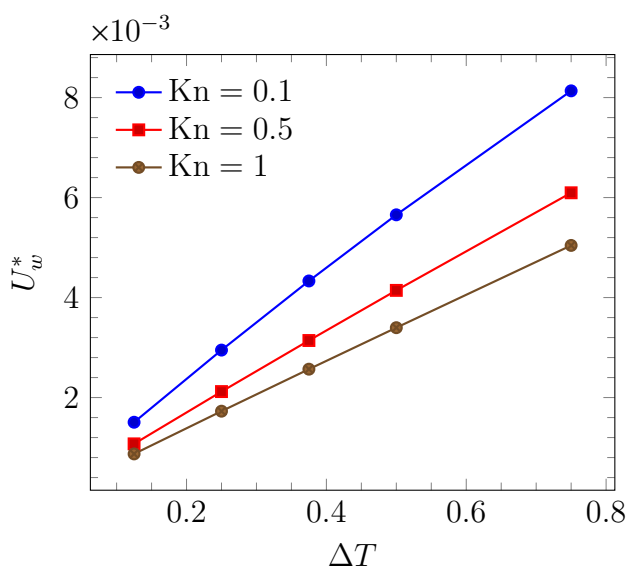

(b)

FIG. 12. Static wall tangential force (a) and shear-free wall velocity (b) against temperature difference. 


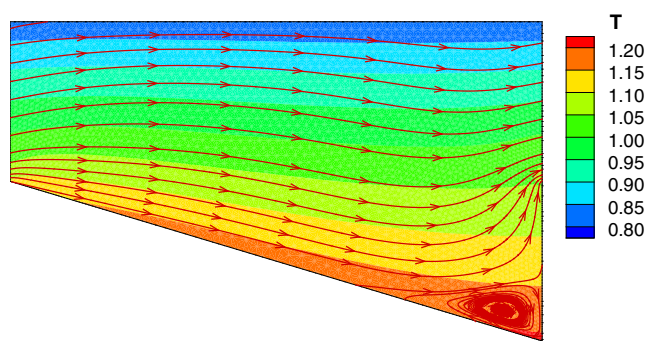

(a)

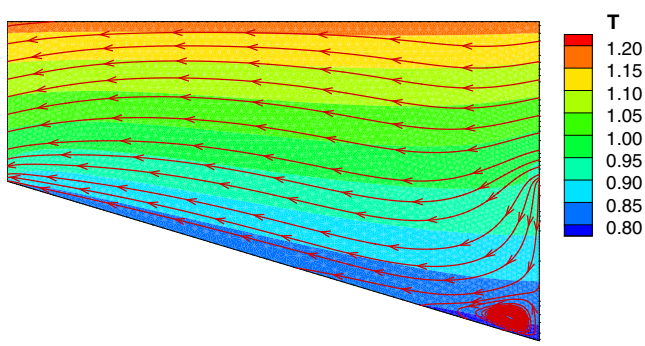

(b)

FIG. 13. Temperature field and streamlines at two temperature configurations. (a) Hot-bottom case $\left(T_{t}=0.75, T_{b}=1.25\right)$. (b) Hot-top case $\left(T_{t}=1.25, T_{b}=0.75\right)$. In both cases, $\mathrm{Kn}=0.1$ and $U_{w}=0$.

mechanical efficiency. The velocity field in shear-free states at $\mathrm{Kn}=0.01,0.1,1$, and 10 are shown in Fig. 11. Comparing Fig. 11 with Fig. 8, we can see when the Knudsen number is small, the flow fields at the bottom half of the channel change only slightly from the static wall states, and the shear flows in the upper half of the channel are balanced by the shear induced by the moving wall. However, at $\mathrm{Kn}=10$ the flow field at shear-free state is very different from that at static wall state. A large vortex occupying almost the whole upper half of the channel develops, even though the overall flow is very weak. Due to the large vortex, the net total mass flux through the channel is almost zero at $\mathrm{Kn}=10$.

\section{Influence of the temperature difference}

We now investigate the influence of the temperature difference between the top wall and the inclined bottom wall. The mean temperature $T_{0}=1 / 2\left(T_{t}+T_{b}\right)$ is fixed, and the wall temperatures $T_{b}$ and $T_{t}$ are adjusted to achieve various temperature difference $\Delta T=T_{b}-T_{t}$. Five different values of $\Delta T$ in the range of $[0.125,0.75]$ are considered. For each case, the SWF and SFV are measured and the results are shown in Fig. 12. Only three Knudsen numbers, i.e., $\mathrm{Kn}=0.1,0.5$, and 1 , are considered, as $F^{*}$ and $U_{w}^{*}$ take their maximum values in this range of $\mathrm{Kn}$, as demonstrated in Fig. 7. From Fig. 12 we can see $F^{*}$ and $U_{w}^{*}$ scale almost linearly with the temperature difference, indicating the strength of thermally induced flow is proportional to the temperature difference.

In the previous analyses, the bottom wall maintains at a higher temperature than the top wall, i.e., $\Delta T>0$. Now we consider the reversed case, namely, a cold-bottom and hot-top configuration $\left(T_{b}=0.75\right.$ and $\left.T_{t}=1.25\right)$. All other parameters are kept the same, i.e., $U_{w}=0$ and $H=B=0.3$. The temperature and flow fields at $\mathrm{Kn}=0.1$ are presented in Fig. 13. It can be seen that the main flow direction in the reversed temperature configuration is reversed accordingly. Both the temperature and the velocity fields have similar structures in the two cases, but the contour levels and the flow direction are reversed. To show the quantitative difference between the two configurations, we plot the shear stress distribution along the top wall in Fig. 14 for the two configurations. The sign of the shear stress for the hot-top case is changed for a better comparison. It can be seen that the local shear stress in the hot-top case is larger than that in the hot-bottom one. This difference can be explained by the temperature dependence of the viscosity because the absolute value of velocity field and hence the shear rate distribution is almost the same in the two configurations. In the hot-top case, the local gas viscosity near the top wall is larger than that in the hot-bottom one due to the much higher local temperature.

\section{Influence of the geometry configuration}

In the previous cases, the geometry parameters are fixed at $H=0.3$ and $B=0.3$. We now study the influence of those geometry parameters to the SWF and the SFV.

First, we fix the ratio of $H$ and $B$ at one but change their absolute values. Five values of $H / L$ will be considered. The SWF and the SFV are shown in Fig. 15. We can see both $F^{*}$ and $U_{w}^{*}$ increase with the increasing of $H$, suggesting greater tangential force can be obtained using a higher ratchet. This trend can be explained as follows. A larger value of $H$ means the inclined bottom wall is steeper and thus the gas temperature gradient near the inclined wall is more significant, which results in a stronger thermal creep flow in the channel.

Then we study the impact of the ratio between $H$ and $B$. The width of the channel, i.e., $H+B$ is fixed, but different values of $B / H$ are used. The SWF and SFV at various values of $B / H$ are shown in Fig. 16 . It can be seen that $B / H$ has a

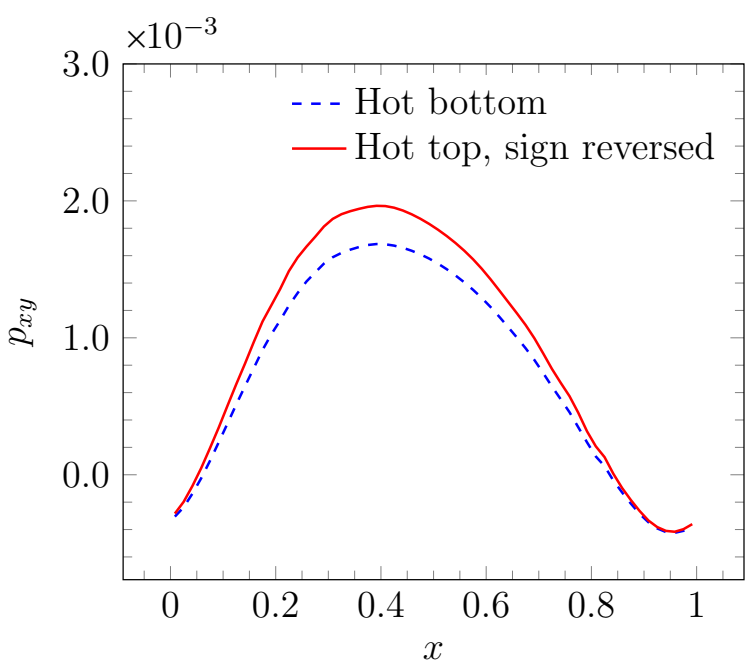

FIG. 14. Shear stress along the top wall at two different temperature configurations. In the hot-bottom case, $T_{t}=0.75$ and $T_{b}=1.25$. In the hot-top case, $T_{t}=1.25$ and $T_{b}=0.75$. In both cases, $\mathrm{Kn}=0.1$ and $U_{w}=0$. 


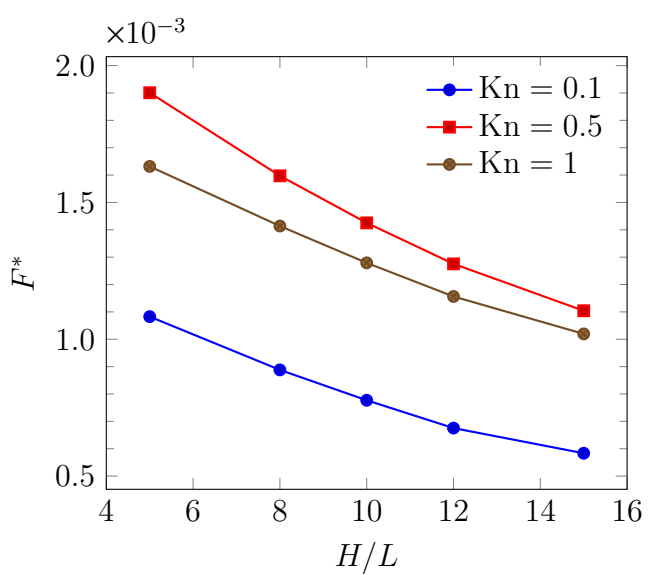

(a)

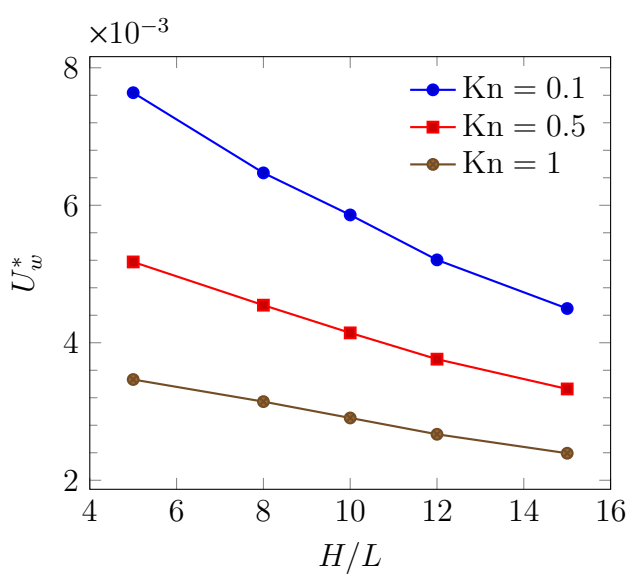

(b)

FIG. 15. Static wall tangential force (a) and shear-free velocity wall velocity (b) as functions of $H / L$ at various Knudsen numbers.

significant influence on the SWF and SFV. Generally, a small value of $B / H$ leads to much larger $F^{*}$ and $U_{w}^{*}$. In the limit of $B / H \rightarrow \infty$, both $F^{*}$ and $U_{w}^{*}$ vanish. This is reasonable since a nearly flat bottom ratchet surface $(B / H \rightarrow \infty)$ will induce a negligible thermal driving effect.

\section{E. Mechanical performance as a heat engine}

We now investigate the mechanical performance and thermal efficiency of the ratchet structure system when it acts as a heat engine. At steady state, the thermal energy is transferred from the hot surface to the cold one, with a net loss which is converted to mechanical work done on the moving top wall. The output power can be calculated as $P=U_{w} F$. As analyzed in Sec. IV B, the tangential force is linearly related to the velocity of the top wall. Then we have $P=-\left(F^{*} / U_{w}^{*}\right) U_{w}^{2}+F^{*} U_{w}$. Using the results from Sec. IV B, we plot the curves of the output power as a function of the top wall velocity in Fig. 17. Similar to the SWF and SFV, the power curves are also affected by the Knudsen number, the temperature difference, and geometric configurations. From Fig. 17, we can observe that the maximum output power and the wall velocity at which the power achieves its maximum change nonmonotonically with the Knudsen number. The maximum power $P_{\max }$ and the corresponding thermal efficiency $\eta_{\max }$ as functions of the Knudsen number are presented in Fig. 18. The maximum thermal efficiency is calculated as $\eta_{\max }=P_{\max } / Q_{\mathrm{h}}$, where $Q_{\mathrm{h}}$ is the total heat flux on the top wall when the output power reaches its maximum. We can assume the top wall velocity has negligible influence on the total heat flux on the walls, which is reasonable since the flow velocity is so small that the temperature field is not affected apparently by the flow of the gas, and subsequently the coupling between the temperature field and the velocity fields is weak. Figure 18 shows that both the maximum output power and the maximum thermal efficiency increase rapidly with the increase of $\mathrm{Kn}$ as $\mathrm{Kn}<0.3$, but then they drop gradually. It is observed that the thermal efficiency is in the order of $0.001 \%$, which is extremely small compared with the Carnot efficiency of an ideal heat engine with the same temperature

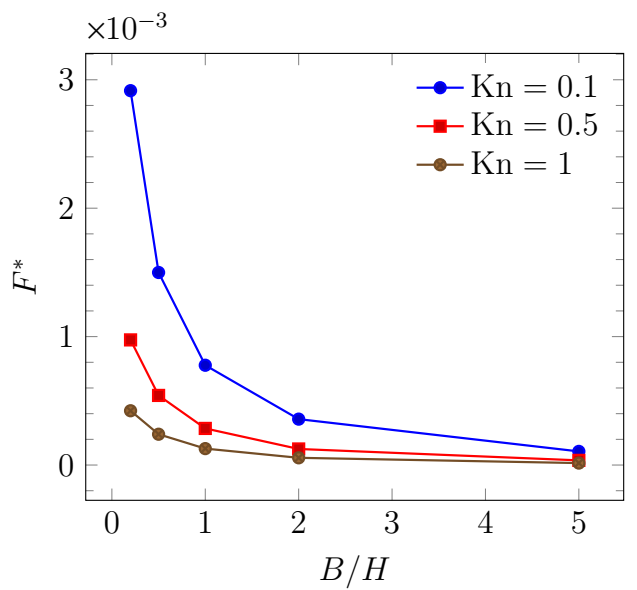

(a)

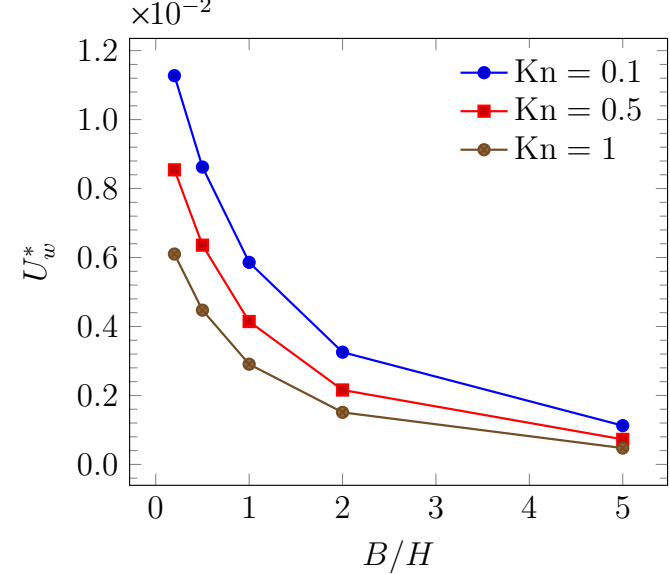

(b)

FIG. 16. Static wall tangential force (a) and shear-free velocity wall velocity (b) as functions of $B / H$ at various Knudsen numbers. 


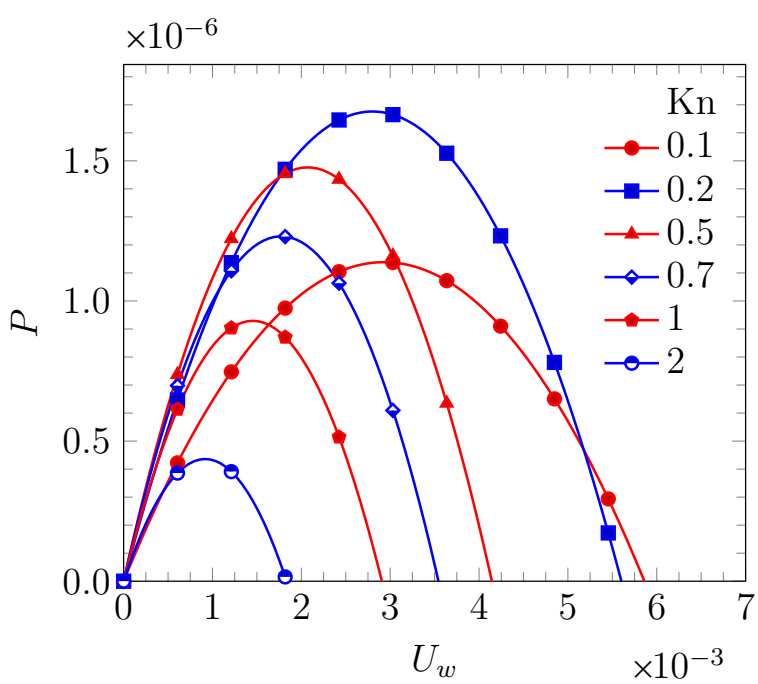

FIG. 17. Dependence of the power output on the wall velocity at various Knudsen numbers.

configuration. This is due to the finite thermal driving effect that the current ratchet structure can generate.

We note that the thermal efficiency here is also much smaller than those obtained in [11], in which the thermal efficiency of a similar structure is analyzed in the free molecular limit. This difference can be explained by the different placements of the specular reflective wall segment and different width and length ratio of the channel. On the one hand, the specular reflective segment is placed vertically in this study, while in [11] the specular reflective segment is placed inclined and the angle of inclination can be adjusted. According to [11], the highest thermal efficiency is obtained with an inclination angle around $20^{\circ}$, and it is much smaller in the limit of $90^{\circ}$ which is corresponding to the vertical configuration in this study. On the other hand, the ratio of width to length, i.e., $H / L$ of the ratchet structure is 0.1 or even smaller in [11]. While in our base configuration, it is 0.3 which indicates a much smaller output power and hence the thermal efficiency according to the observations in Sec. IV D.

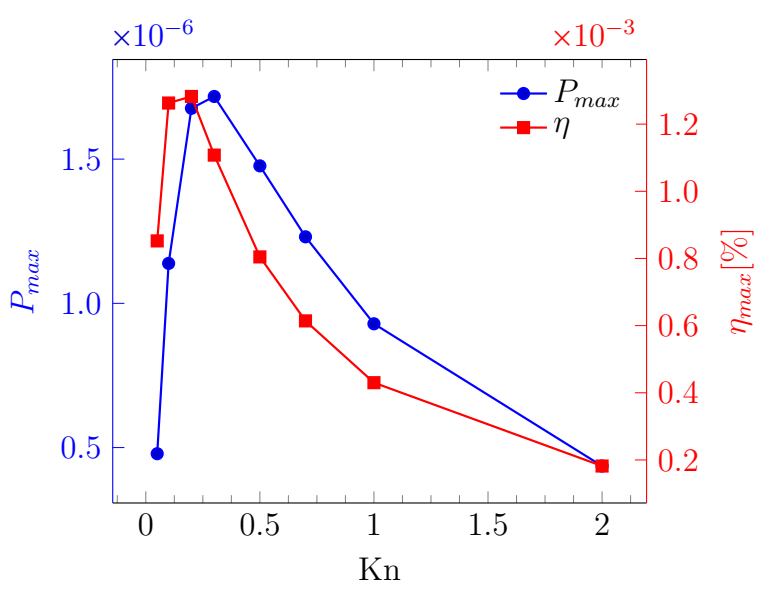

FIG. 18. Maximum power output and thermal efficiency.
The effect of temperature difference to the maximum output power and thermal efficiency can be analyzed using the results in Sec. IV C. Because both the SWF and the SWF scale nearly linearly with the temperature difference, the output power is expected to scale quadratically with the increase of the temperature difference. The thermal efficiency will increase linearly with the increase of the temperature since the heat flux will also scale linearly at the same time.

\section{CONCLUDING REMARKS}

In the present study, motivated by the potential application of thermal creep flow as an energy harvesting device, the thermal and boundary driven gas flows in a microchannel with ratchet surface in a wide range of operating conditions have been invested. The tangential force acting on the channel wall at different wall velocity are obtained by deterministically solving the Boltzmann model equation using the recently proposed discrete unified gas-kinetic scheme. It is found the tangential force changes linearly with the wall velocity, and the parameters characterizing the linear relations are obtained in various operating conditions. The tangential force on the static wall and the top wall velocity at shear-free state achieve their maximum values in the Knudsen number range of $[0.1,1]$ and are found to be influenced linearly by the imposed wall temperature difference. The relative height of the ratchet structures has a significant influence on the thermal driving effect, namely a higher ratchet structure leads to a larger tangential force.

The mechanical power output and the thermal efficiency when the system works as a heat engine are also measured based on the relation between the tangential shear force and the wall velocity. It is found that both of them can achieve the maximum values in the early transition regime and are significantly larger than those in the free molecular regime.

The thermal driving effect produced by the current inclined diffusive reflective wall and vertical specular reflective wall configuration is relatively small compared with the inclined specular reflective wall configurations in previous studies. Future study should consider more general geometry configurations to improve the thermal efficiency.

\section{ACKNOWLEDGMENTS}

This study was supported by the National Key Research and Development Plan (Grant No. 2016YFB0600805).

\section{APPENDIX: DISCRETE UNIFIED GAS-KINETIC SCHEME}

The numerical scheme used to solve the Boltzmann model equation is the discrete unified gas-kinetic scheme (DUGKS) [18,19,22], which is an explicit finite-volume scheme for the discrete velocity Boltzmann model equation. We only briefly mention the major steps of this method, since the method presented here is essentially the same as unstructured mesh-based DUGKS in [22]. Moreover, the method has been implemented as an open source solver in the framework of OpenFOAM [24]. 
The governing equation, i.e., Eq. (9), is first discretized in the velocity space with a chosen two-dimensional discrete velocity grid $\left\{\boldsymbol{\xi}_{\alpha}, \alpha=1,2, \ldots, M\right\}$,

$$
\frac{\partial \Phi_{\alpha}}{\partial t}+\xi_{\alpha, x} \frac{\partial \Phi_{\alpha}}{\partial x}+\xi_{\alpha, y} \frac{\partial \Phi_{\alpha}}{\partial y}=-\frac{1}{\tau}\left[\Phi_{\alpha}-\Phi_{\alpha}^{S}\right] \equiv \Omega_{\alpha},
$$

where $\Phi_{\alpha}$ and $\Phi_{\alpha}^{S}$ are the distribution function and equilibrium distribution function with discrete velocity $\xi_{\alpha}$. Equation (A1) is then discretized in the spatial space with the following cell centered finite-volume scheme [19]:

$$
\begin{aligned}
\Phi_{\alpha, k}^{n+1}-\Phi_{\alpha, k}^{n}+\frac{\Delta t}{\left|V_{k}\right|} \mathcal{F}_{\alpha, k}^{n+1 / 2} & =\frac{\Delta t}{2}\left[\Omega_{\alpha, k}^{n+1}+\Omega_{\alpha, k}^{n}\right], \\
k & =\{1,2, \ldots, N\},
\end{aligned}
$$

where $\Phi_{\alpha, k}^{n}$ is the cell averaged value $\Phi_{\alpha}$ of cell $k$ at time level $t^{n},\left|V_{k}\right|$ is the volume of the cell $k, N$ is the total number of cells, and $\Delta t=t^{n+1}-t^{n}$ is the time step. The flux $\mathcal{F}_{\alpha, k}^{n+1 / 2}$ is evaluated at the middle time step by

$$
\mathcal{F}_{\alpha, k}^{n+1 / 2}=\sum_{l} \boldsymbol{\xi}_{\alpha} \cdot \boldsymbol{S}_{k, l} \Phi_{\alpha, k, l}^{n+1 / 2},
$$

where $\boldsymbol{S}_{k, l}$ is the surface vector of face $l$ belonging to cell $k$, and $\Phi_{\alpha, k, l}^{n+1 / 2}$ is the face centered distribution function of face $l$ at the middle time step. The main idea behind DUGKS is that the $\Phi_{\alpha}^{n+1 / 2}$ at cell face center is constructed in a physical way by solving the governing equation locally around the cell face from time $t^{n}$ to time $t^{n+1 / 2}$. The evolution equation for $\Phi_{\alpha}$ at the cell face center $\boldsymbol{x}_{f}$ is [19]

$$
\begin{aligned}
& \Phi_{\alpha}^{n+1 / 2}\left(\boldsymbol{x}_{f}\right)-\Phi_{\alpha}^{n}\left(\boldsymbol{x}_{f}-\boldsymbol{\xi}_{\alpha} \Delta t / 2\right) \\
& \quad=\Delta t / 4\left[\Omega_{\alpha}^{n+1 / 2}\left(\boldsymbol{x}_{f}\right)+\Omega_{\alpha}^{n}\left(\boldsymbol{x}_{f}-\boldsymbol{\xi}_{\alpha} \Delta t / 2\right)\right] .
\end{aligned}
$$

In the above equation, the distribution functions at $\boldsymbol{x}_{f}-$ $\xi_{\alpha} \Delta t / 2$ are interpolated linearly from the nearby cell centered distribution functions.

The implicitness of Eq. (A2) and Eq. (A4) is eliminated by introducing two variable transformations [19],

$$
\tilde{\Phi}_{\alpha}=\Phi_{\alpha}-\Delta t / 2 \Omega_{\alpha}, \quad \bar{\Phi}_{\alpha}=\Phi_{\alpha}-\Delta t / 4 \Omega_{\alpha} .
$$

In the actual implementation, $\tilde{\Phi}_{\alpha}$ is tracked instead of the original $\Phi_{\alpha}$. The macrovariables at each time step are evaluated by taking moments of $\tilde{\Phi}_{\alpha}$ as follows:

$$
\begin{aligned}
\rho & =\sum_{\alpha} w_{\alpha} \tilde{g}_{\alpha}, \quad \rho U_{i}=\sum_{\alpha} w_{\alpha} \xi_{\alpha, i} \tilde{g}_{\alpha}, \\
\rho E & =\frac{1}{2} \sum_{\alpha} w_{\alpha}\left[\left(\xi_{\alpha, x}^{2}+\xi_{\alpha, y}^{2}\right) \tilde{g}_{\alpha}+\tilde{h}_{\alpha}\right], \\
q_{i} & =\frac{2 \tau}{2 \tau+\Delta t \operatorname{Pr}} \tilde{q}_{i}, \quad \text { with } \\
\tilde{q}_{i} & =\frac{1}{2} \sum_{\alpha} w_{\alpha} c_{\alpha, i}\left\{\left[c_{\alpha, x}^{2}+c_{\alpha, y}^{2}\right] \tilde{g}_{\alpha}+\tilde{h}_{\alpha}\right\}, \\
p_{i j} & =\frac{2 \tau}{2 \tau+\Delta t} \tilde{p}_{i j}, \quad \text { with } \tilde{p}_{i j}=\sum_{\alpha} w_{\alpha} c_{\alpha, i} c_{\alpha, j} g_{\alpha} .
\end{aligned}
$$

[1] Y. Sone, Molecular Gas Dynamics: Theory, Techniques, and Applications (Birkhäuser, Boston, 2007).

[2] W. Crookes, Philos. Trans. R. Soc. London 164, 501 (1874).

[3] A. Passian, A. Wig, F. Meriaudeau, T. L. Ferrell, and T. Thundat, J. Appl. Phys. 92, 6326 (2002).

[4] A. Ketsdever, N. Gimelshein, S. Gimelshein, and N. Selden, Vacuum 86, 1644 (2012).

[5] N. Selden, C. Ngalande, N. Gimelshein, S. Gimelshein, and A. Ketsdever, J. Fluid. Mech. 634, 419 (2009).

[6] S. Chen, K. Xu, and C. Lee, Phys. Fluids 24, 111701 (2012).

[7] J. Nabeth, S. Chigullapalli, and A. A. Alexeenko, Phys. Rev. E 83, 066306 (2011).

[8] S. Taguchi and K. Aoki, Phys. Rev. E 91, 063007 (2015).

[9] J. C. Maxwell, Philos. Trans. R. Soc. London 170, 231 (1879).

[10] A. A. Donkov, S. Tiwari, T. Liang, S. Hardt, A. Klar, and W. Ye, Phys. Rev. E 84, 016304 (2011).

[11] T. Baier, J. Dölger, and S. Hardt, Phys. Rev. E 89, 053003 (2014).

[12] A. Würger, Phys. Rev. Lett. 107, 164502 (2011).
[13] H. Linke, B. J. Alemán, L. D. Melling, M. J. Taormina, M. J. Francis, C. C. Dow-Hygelund, V. Narayanan, R. P. Taylor, and A. Stout, Phys. Rev. Lett. 96, 154502 (2006).

[14] G. Lagubeau, M. Le Merrer, C. Clanet, and D. Quéré, Nat. Phys. 7, 395 (2011).

[15] J. Ok, E. Lopez-Oña, D. Nikitopoulos, H. Wong, and S. Park, Microfluid Nanofluid 10, 1045 (2011).

[16] S. Hardt, S. Tiwari, and T. Baier, Phys. Rev. E 87, 063015 (2013).

[17] J. Chen, L. Baldas, and S. Colin, Vacuum 109, 294 (2014).

[18] Z. Guo, K. Xu, and R. Wang, Phys. Rev. E 88, 033305 (2013).

[19] Z. Guo, R. Wang, and K. Xu, Phys. Rev. E 91, 033313 (2015).

[20] G. A. Bird, Molecular Gas Dynamics and the Direct Simulation of Gas Flows (Clarendon Press, Oxford, 1994).

[21] E. M. Shakhov, Fluid Dyn. 3, 95 (1968).

[22] L. Zhu, Z. Guo, and K. Xu, Comput. Fluids 127, 211 (2016).

[23] T. J. Scanlon, E. Roohi, C. White, M. Darbandi, and J. M. Reese, Comput. Fluids 39, 2078 (2010).

[24] L. Zhu, S. Chen, and Z. Guo, Comput. Phys. Commun. 213, 155 (2017). 